Basrah Journal Of Surgery
Editorial

Bas J Surg, December, 24, 2018

\title{
IS THERE A “FREE SIZE” IN SURGICAL PRACTICE?
}

"Not all that glitters is gold"

Geoffrey Chaucer

\section{Thamer A Hamdan}

MB,ChB, FRCS, FICS, FACS, Professor of Orthopedic Surgery, Chancellor of Basrah University, Basrah, IRAQ.

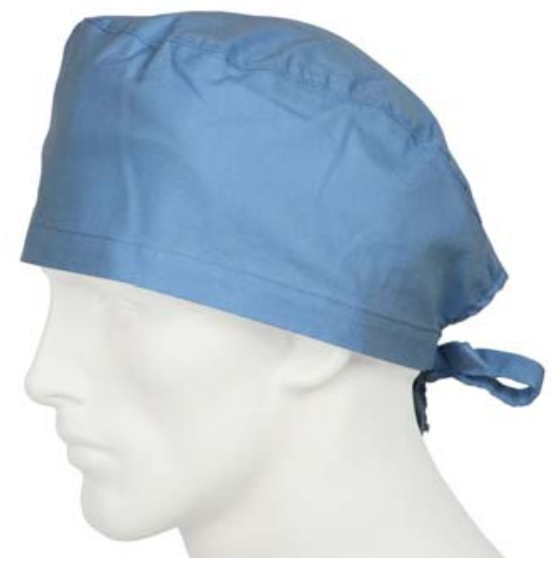

S everal parameters should seriously be considered in, almost, every pathology before $\checkmark$ drawing the line of treatment. Patients are not alike even when they are twins. Individual variation is a reality that should be taken in consideration.

On top of this, comes the response to pain. The best example is the delivery of a baby; while one lady shouts loudly, another is very quiet. Some consider the pain stimulus as intense, because of psychogenic factors, while others show a calm response for the same condition.

A well-known fact is the individual variation in response to medication, like nonsteroidal anti-inflammatory drugs which might be very effective for $\mathrm{Mr}$. X, but not for Mr. Y. Similarly, the healing power, immunity status and even the creation of some pathology like stone formation, are not homogeneous. I noticed, in my career, patients who are good callus formers, while others are poor callus formers.

Same pathology may have different clinical presentation in relation to body distribution and even the type of inflammatory response. A recognized fact is the variation in geographical distribution of disease between countries.

Other factors which are related to each individual patient and should be kept in mind, include the mental and psychosocial status. It is well-known that a depressed patient has low pain threshold.

Patient cooperation to the planned therapeutic regimen is also very variable. Some habits and hobbies may play a role in the success of disease treatment. Smoking, for example, can influence healing and recovery of a disease, and can increase the incidence of infection, so as alcohol consumption. The degree of body and cloth cleanliness is another vital point to be considered. 
I have noticed a definite individual variation in the metabolic response to injury. In addition, the financial status of each individual patient may play a role in the selection of treatment regimen. The availability of a particular medication in the local market and finding the alternative is a point to be considered seriously.

Building on the points mentioned above, there is nothing to be called, as in the clothes, a "free size" treatment; whether surgical or medical treatments, both should be tailored to each individual patient. There is no one standard treatment for all patients. Patient's status needs to be analyzed, taking in consideration all the above cited factors before determining the line of treatment.

Lastly, I prefer to mention two very informative examples of treatment individualization; one is the ideal surgical versus medical treatment for diabetes, and the second is finding the best surgical or conservative treatment for the same fracture.

To sum up, there is no one "free size" treatment that fits all patients in surgical or medical practices. Finally, I must say that nothing new was mentioned in this editorial except what was forgotten.
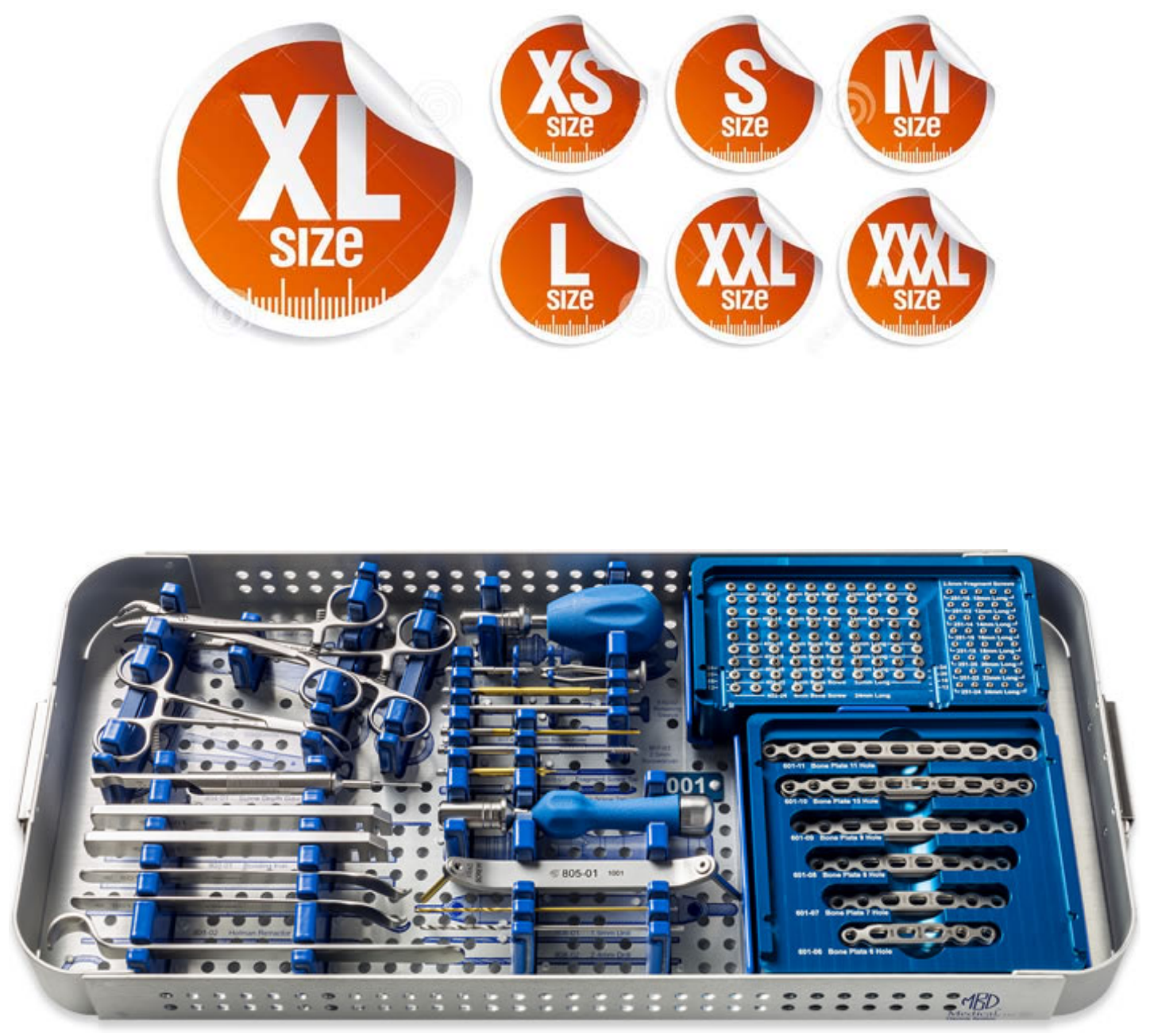